\title{
Canine maxillaire et prothèse amovible partielle
}

\section{RÉSUMÉ}

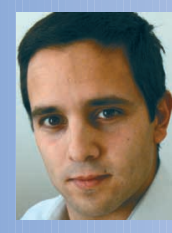

Antoine KAROUBI

Ancien Assistant des Universités, Ancien Assistant Hospitalier,

26, avenue de Paris,

92320 Châtillon.

\section{Sarah BUECHE}

Ancienne attachée hospitalo-universitaire,

Université René Descartes Paris $V_{\text {, }}$

1 , rue Maurice Arnoux,

92120 Montrouge.
Clé de voûte du maxillaire, la canine supérieure assure à la fois un rôle esthétique dans le secteur antérieur et un rôle fonctionnel car faisant partie intégrante du guidage antérieur avec les incisives.

Son remplacement en cas de perte ou d'absence doit être abordé de façon multidisciplinaire.

La prothèse amovible partielle à châssis métallique peut se présenter comme une thérapeutique de choix grâce à son économie tissulaire et à son faible coût financier.

Deux cas cliniques illustrent le remplacement de la canine d'une part grâce à la PAP conventionnelle et d'autre part grâce à la prothèse composite, afin de concilier esthétique, confort, intégration occlusale de la prothèse et réhabilitation globale du patient.
- guidage canin

- prothèse amovible partielle

prothèse composite 


\section{Introduction}

$>$

La canine, par sa situation antérieure

(visible et facile d'accès au brossage), par son anatomie coronaire, par sa grande longueur de racine, et par son parondonte particulièrement robuste au niveau de la bosse canine, est souvent la dernière dent à quitter la cavité buccale.

Son absence, ou sa perte, est donc rare. Les étiologies sont essentiellement les agénésies ou les traumatismes[1, 2].

L'indication d'une restauration par prothèse amovible partielle (PAP) doit être le résultat d'une analyse préprothétique globale et multidisciplinaire, tenant compte des doléances du patient relevées lors de l'entretien clinique[3, 4]. La PAP est souvent I'ultime solution thérapeutique possible quand la prothèse conjointe ou la prothèse implanto-portée ont été écartées, notamment pour les édentements maxillaires de grande étendue.

Cette thérapeutique présente des difficultés esthétiques et biomécaniques spécifiques que nous illustrerons à travers deux situations cliniques.

\section{Spécificités thérapeutiques}

\section{Forces et faiblesses}

des traitements

par PAP

\section{dans ces situations}

Celles-ci sont fonction de l'étendue de l'édentement - unitaire ou plural - et de sa situation - antérieur ou postérieur -[4,5].

\section{$>$ Avantages}

- Pratiquement jamais de contre-indication.

- Coût financier faible.

- Temps de traitement court.

- Coût tissulaire faible (améloplasties).

- Esthétique : liberté dans le placement de la canine, possibilité de compenser les pertes du volume alvéolaire par une fausse gencive, notamment de reconstituer une bosse canine.

\section{> Inconvénients}

- Psychologique : les surcontours générés par les éléments métallique du châssis sur les dents d'appui peuvent être ressentis avec désagrément par le patient.

- Esthétique : visibilité de certaines parties du châssis (crochets, taquets...) de la fausse gencive...

\section{Difficultés esthétiques}

Le choix de la forme, de la teinte et de la position de la canine joue un rôle majeur dans le caractère du sourire, reflet de la personnalité du patient[5].

Dans une situation d'édentement antérieur, la PAP participe au soutien des lèvres donc à l'esthétique exobuccale et à l'expression faciale. Chez un patient édenté de longue date, présentant une fonte ostéo-muqueuse impor- 
tante, la canine prothétique et la fausse gencive doivent simuler la bosse canine pour rétablir un soutien harmonieux de la lèvre, ce qui peut parfois réduire le sillon naso-génien et gommer certaines rides. À l'inverse, lorsque la perte dentaire est récente, la persistance $d^{\prime} u n$ rempart osseux peut contre-indiquer l'apposition de fausses gencives. II faut alors réaliser des dents ajustées, ce qui peut s'avérer délicat à harmoniser avec les autres dents antérieures, notamment dans une situation de sourire gingival.

Dans un édentement postérieur incluant la canine, la difficulté esthétique est double :

- si pour un édentement encastré de courte à moyenne étendue, le crochet antérieur peut parfois être supprimé, pour un édentement de grande étendue ou en extension, le recours à la prothèse composite évite de placer un crochet disgracieux et peu efficace sur une incisive. Couronner une ou plusieurs dents du secteur incisif permet d'y dissimuler des éléments de rétention comme des attachements. Ces traitements plus complexes nécessitent une parfaite maîtrise de la chaîne prothétique et une bonne communication et coordination avec les différents prothésistes de laboratoire ;

- le point de contact entre la dernière dent naturelle et la première dent prothétique crée une zone d'ombre dans l'embrasure qui dessine un triangle noir, à l'endroit même où commence la fausse gencive. Cette transition, délicate à gérer esthétiquement, peut trahir la présence d'une PAP.

\section{Difficultés fonctionnelles}

La canine joue un rôle majeur lors de la mastication, dans les mouvements d'incision et de dilacération. Associée aux incisives, elle parti- cipe au guidage antérieur lors de la propulsion pour créer une désocclusion postérieure. Lors des mouvements de latéralité, on parle de "fonction canine» quand celle-ci assure seule le guidage mandibulaire et de "fonction de groupe» lorsqu'elle est associée à d'autres dents adjacentes[6].

Les forces appliquées à la canine lors de cette fonction occlusale ont souvent une forte composante horizontale qui est très déstabilisante pour une PAP.

De plus, par sa situation à l'angle de l'arcade, jonction entre le secteur antérieur et postérieur, la canine se trouve exinscrite des polygones de sustentation et de rétention de la PAP. C'est une situation comparable aux édentements en extension où la prothèse subit des mouvements déstabilisants de translation et de rotation lors de la mastication.

C'est pourquoi, pour garantir l'équilibre de la prothèse, assurer la stabilité occlusale et préserver la santé des structures neuro-musculoarticulaires, le praticien doit respecter certaines règles :

- respecter les principes de conception des prothèses à châssis métallique ;

- rétablir de préférence une fonction de groupe ;

- obtenir un guidage symétrique ;

- créer une désocclusion postérieure minimale et homogène $[5,7]$.

L'architecture de la prothèse ainsi que le choix du schéma occluso-fonctionnel est le résultat d'une analyse globale de la situation clinique et des modèles d'étude montés en articulateur. Il faut tenir compte de nombreux paramètres: étendue et type de l'édentement (encastré ou terminal), nature de l'antagoniste, mode de restauration prothétique choisi, valeur des dents restantes et de leur parodonte, etc. 


\section{Illustrations cliniques}

Cas clinique $\mathrm{n}^{\circ} 1$ :

Édentement unitaire

de canine

Dans cette première situation clinique il s'agit d'une patiente de 58 ans qui consulte pour le renouvellement de sa PAP en résine remplaçant la canine maxillaire droite absente «depuis toujours».

À l'examen endobuccal, on note l'absence de 13 sur l'arcade et le délabrement de 15 nécessitant une restauration par prothèse fixée (fig. 1 a et b).

L'examen tomodensitométrique révèle une canine incluse en mésioversion coronaire mar- quée, la couronne étant palatine en rapport étroit avec les faces palatines de 11 et 12, la racine en contact avec l'apex de 14. L'inclusion et la situation de 13 expliquent la linguomésio-version de 12, I'usure de son bord libre donne une impression de cuspide et une apparence de canine.

\section{> Propositions thérapeutiques}

En raison de l'inclusion basse de 13, la traction orthodontique initialement envisagée et la solution implantaire s'avèrent impossibles.

Une reconstitution par bridge collé est écartée en raison du risque important de décollement en rapport avec la forte sollicitation occlusale lors des mouvements de latéralité
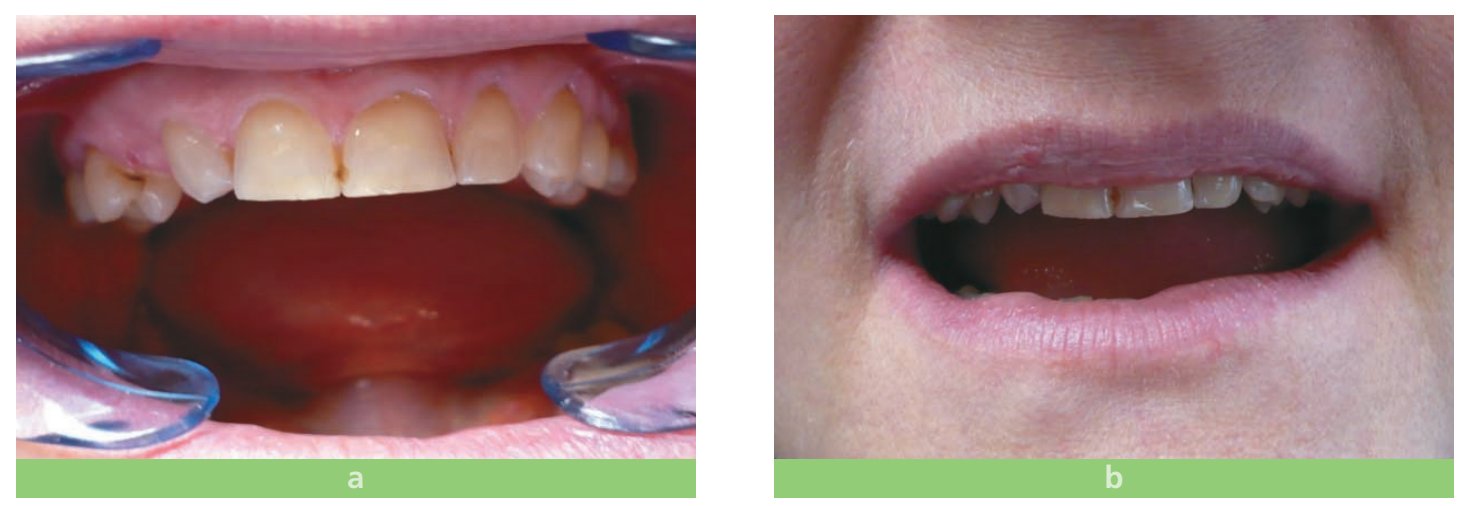

Fig. 1 a et b Vues endobuccales sans prothèse.

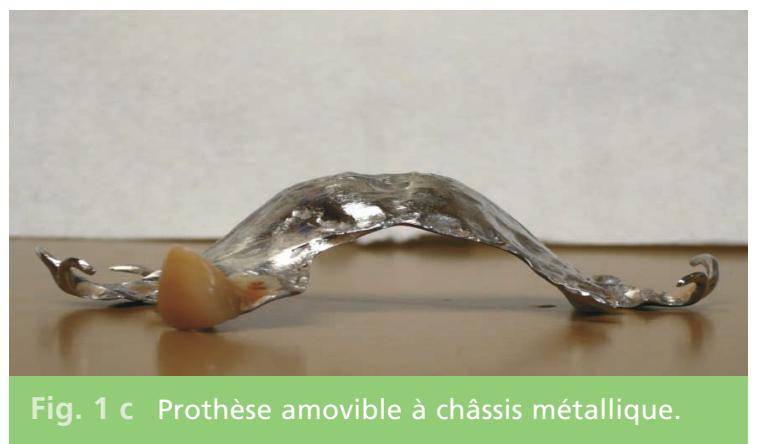


et de la faible hauteur coronaire des piliers 12 et 14 .

Enfin, le bridge conventionnel est refusé par la patiente qui souhaite conserver 12 et 14 intactes.

La prothèse composite est alors proposée : elle comprend une couronne céramo-métallique fraisée sur 15 et une PAP à châssis métallique remplaçant 13 (fig. 1 c). Cette solution est facilement acceptée par la patiente qui portait déjà une prothèse amovible.

\section{Conception}

Dans cette situation encastrée, pour respecter les principes de conception des PAP à châssis métallique, il faut disposer de part et d'autre de l'édentement des potences courtes et rigides en direction d'un appui cingulaire sur 12 et d'un taquet occlusal mésial sur 14 .

En ce qui concerne la rétention, pour des raisons esthétiques, les crochets cavaliers (ou de Bonwill) sont placés plus distalement entre 15 et 16 d'un côté et 25 et 26 de l'autre.

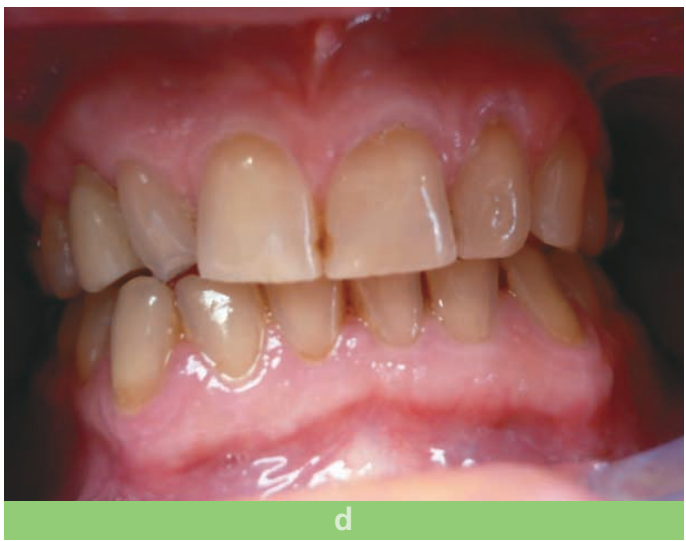

\section{$>$ Avantages}

Contrairement à son ancienne prothèse en résine à recouvrement muqueux maximum, cette PAP à châssis métallique présente des appuis occlusaux, est décolletée et plus rigide. Elle est donc moins délétère pour le parodonte des dents restantes.

De plus, cette solution est beaucoup moins délabrante puisque la seule préparation consiste à réaliser des améloplasties notamment pour le passage des crochets cavaliers.

\section{$>$ Impératifs}

Pour plus de discrétion et une meilleure intégration esthétique, il n'a pas été réalisé de fausse gencive en vestibulaire de 13 (fig. $1 \mathrm{~d}$ et e).

Lors des mouvements de diduction, la 13 s'intègre dans les fonctions de groupe droite et gauche de la patiente qui sont conservées.

Des contrôles réguliers et une bonne maintenance seront recommandés afin d'assurer la pérennité du résultat.

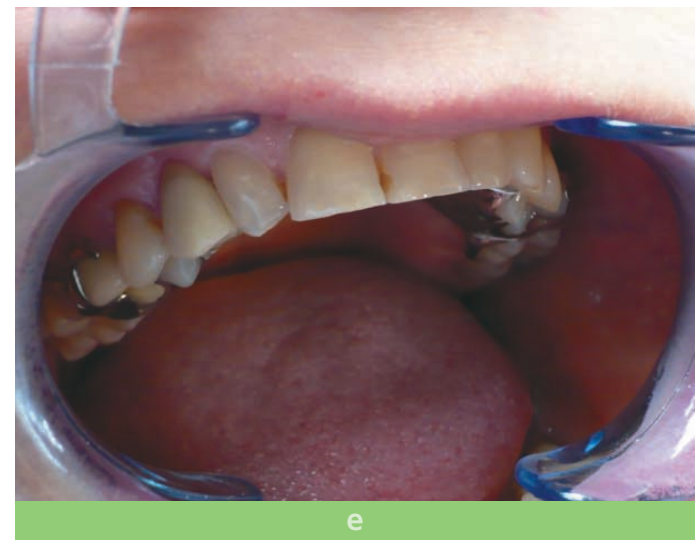


Cas $n^{\circ} 2$ :

\section{Prothèse composite[8]}

Dans cette seconde situation clinique, on note à droite un édentement encastré avec absence de la canine, tandis qu'à gauche ne subsistent dans le secteur cuspidé que les racines de 25 et 27 (fig. 2 a et b). La réhabilitation prothétique comporte un bridge antérieur avec une canine en extension, support d'un attachement intracoronaire de semi-précision, une couronne fraisée sur la 17 et deux chapes d'appui à tenon radiculaire sur la 25 et la 27 (fig. 2 c, d et e).

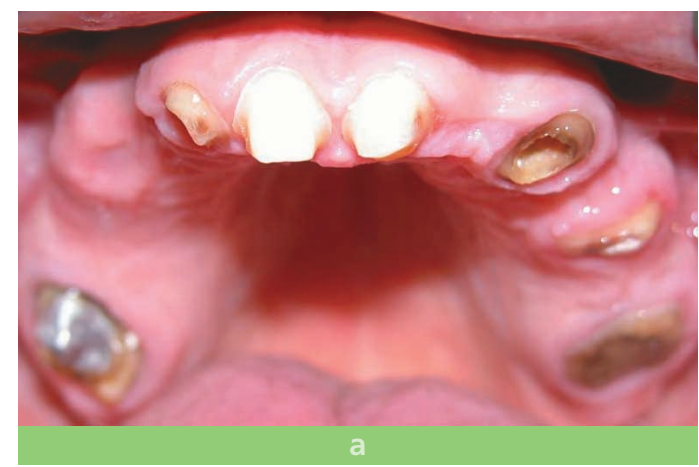

$>$ Conception $[8,9]$

- La canine en extension est permise par le grand arc de solidarisation antérieur.

- La situation encastrée de la selle droite est un élément favorable car elle limite ses possibilités de mouvement.

- La présence des chapes sous-prothétiques du côté gauche empêche l'enfoncement distal de la selle et limite les possibilités de rotation autour d'un axe oblique joignant 17 à 23 .

- L'attachement axial sur la 25 (fig. 2 f) majore la rétention obtenue par une seconde glissière sur la 22 et permet de s'affranchir de la présence d'un crochet sur la 23.

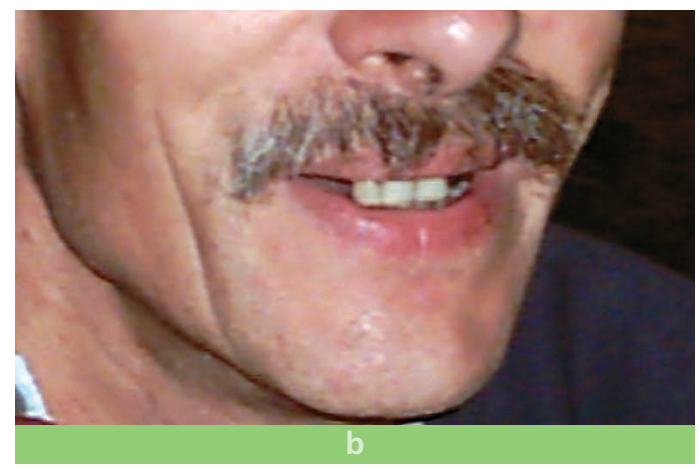

\section{Fig. 2 a et b Situation initiale.}
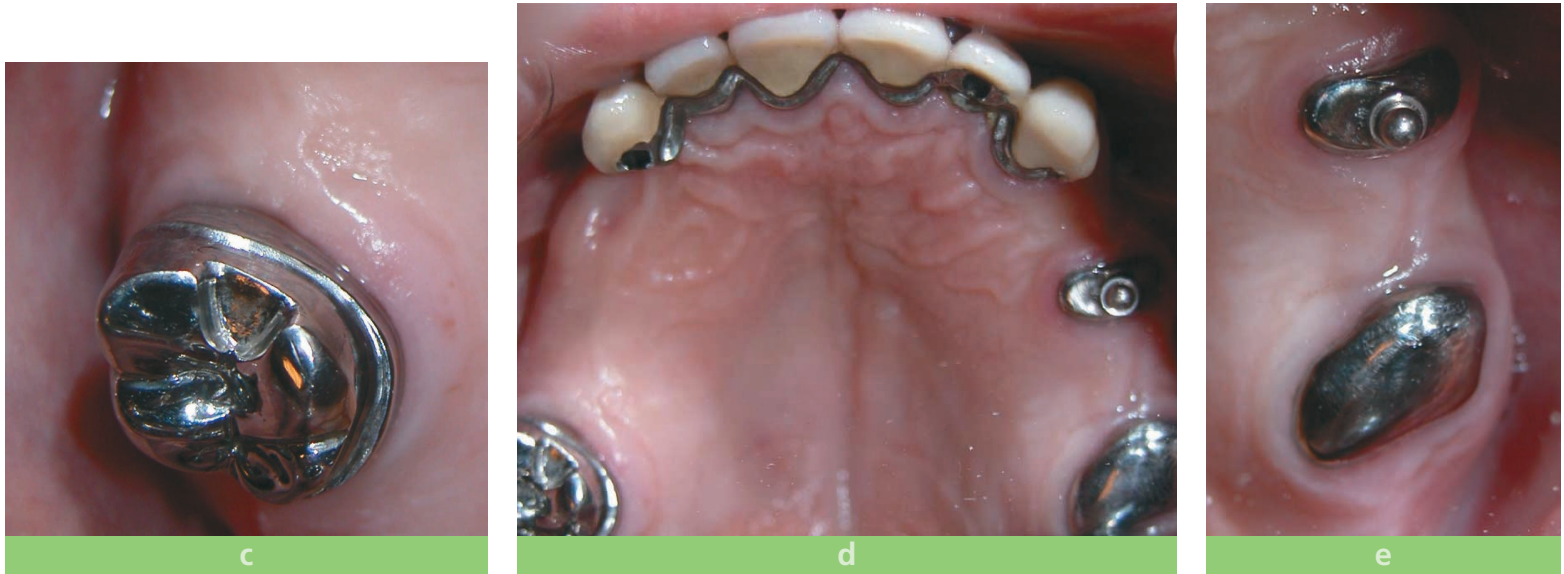

Fig. 2 c, d et e Éléments de prothèse fixée. 


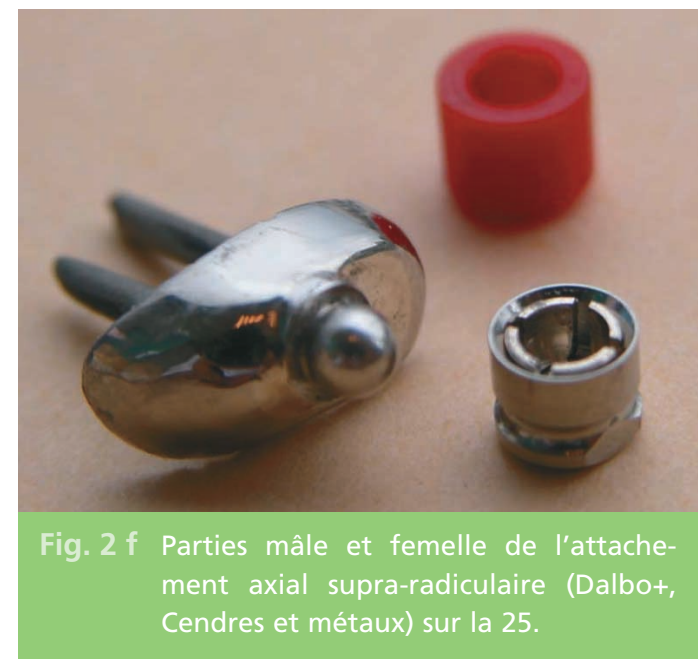

\section{$>$ Avantages}

- Esthétique :

- absence de visibilité des moyens de rétention ;

- le début de la fausse gencive ainsi que le «triangle noir» associé sont repoussés plus distalement.

- Mécanique : maîtrise des mouvements de rotation et de translation grâce aux attachements glissières et aux fraisages.

- Psychologique :

- diminution de la sensation d'encombrement grâce aux fraisages qui intègrent la barre cingulo-coronaire et les bras de réciprocité d'action des crochets ;

- Persistance de la totalité du bloc incisivocanin lorsque le patient enlève sa PAP.

\section{> Impératifs}

La canine en extension doit être déchargée de tout guidage en propulsion et latéralité. L'occlusion dynamique est assurée par des fonctions de groupes. Les contrôles et la maintenance doivent être réguliers : par exemple, une résorption de la crête de secteur 1 , non compensée par une réfection de base, ferait porter toute la charge occlusale à la 17 et la 13, ce qui compromettrait son pronostic (fig. $\mathbf{2}$ g, h, i et j).

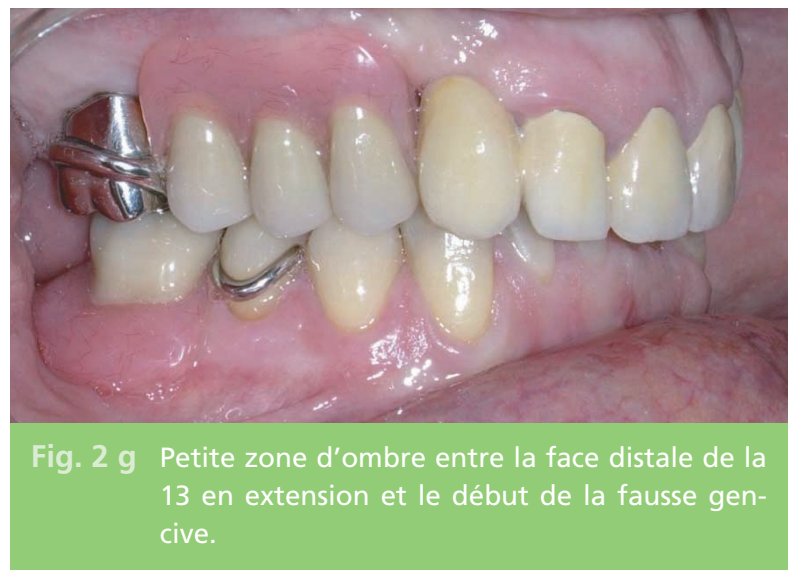



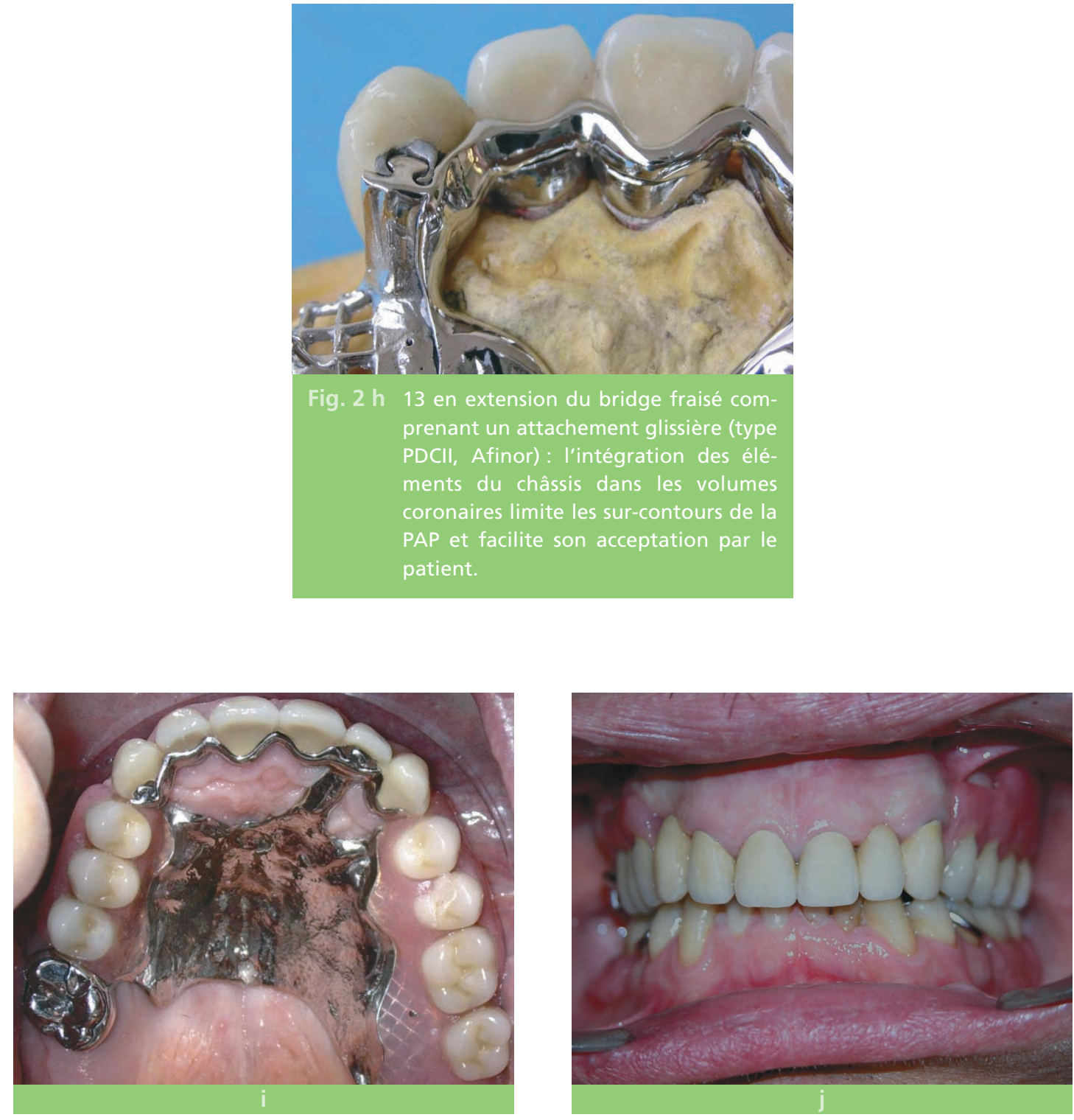

Fig. 2 i et j Vue globale de la réhabilitation prothétique.

Si l'absence de la canine pose des problèmes esthétiques et fonctionnels particuliers, la PAP peut souvent apporter des solutions. Bien qu'une prothèse amovible soit souvent difficile à accepter psychologiquement et à intégrer d'une façon esthétique, la prothèse composite permet de minimiser ces désagréments.
Le succès de ces thérapeutiques nécessite la prise en considération des doléances du patient, une parfaite maîtrise des principes de conception et de la chaîne prothétique ainsi que l'instauration d'une maintenance régulière à long terme afin d'assurer la pérennité de la prothèse. 


\section{Bibliographie}

1. Marcus SE, Drury TF, Brown LJ, Zion GR. Tooth retention and tooth loss in the permanent dentition of adults: United States, 1988-1991.

J Dent Res 1996;75: Spec $n^{\circ}$ 684-695.

2. Steele JG, Treasure E, Pitts NB, Morris J, Bradnock G.

Total tooth loss in the United Kingdom in 1998 and implications for the future. British Dental Journal 2000;189: ${ }^{\circ} 11$.

3. Schittly J. Traitement des édentements antérieurs. Réalités cliniques 1998;9(4):423-433.

4. Cherfane $P$, Tremoureux A, Fromentin O, Tavernier B.

Absence des canines maxillaires permanentes. Stratégie thérapeutique. Cah Prothèse 2007;138:13-21.

5. Begin M, Mollot Ph. Traitement par prothèses composites d'un édentement de classe I bimaxillaire avec absence d'une canine.

Cah Prothèse 1995;90:7-17.

6. Laluque JF, Brocard D. La canine. Fonctions, dysfonction et parafonction. Titane Vol 2007;4(3):5-6.

7. Cheylan JM, Buch D. Couronnes fraisées. Principes de conception. Cah Prothèse 2002;119:59-65.

8. Karoubi A.

Traitement par prothèse composite : Transfert des données esthétiques. P 2007;113:11-14.

9. Amzalag $\mathrm{G}$, Batarec $\mathrm{E}$, Schoendorff R, Buch $D$, Assemat-Tessandier $\mathrm{X}$. Prothèses supraradiculaires : "overdentures". Cah Prothèse 1988.

\section{SUMMARY}

\section{Maxillary canine and removable partial prostheses}

Antoine KAROUBI

Sarah BUECHE

\section{Keywords}

- canine guidance

- removable partial prostheses

- composite prostheses
Important part of maxilla, canine provides both an aesthetic role in the anterior area and a functional role because of its part in the anterior guidance with incisive.

Canine replacement, in case of loss or absence, must be thought in all the ways of our discipline.

Removable partial prostheses can be chosen as a therapy thanks to tissular economy allowed and its low cost.

Two clinical cases show the canine replacement on the one hand with a conventional removable partial prosthesis and on the other hand with a composite prosthesis, in order to get aesthetics, comfort, occlusal prosthetic integration and global rehabilitation of the patient. 


\title{
Les Archives historiques du Rouergue et la Société des lettres, sciences et arts de l'Aveyron \\ présentent
}

\section{La Recherche sur la vraye anathomie des dents, nature et propriétés d'icelles}

\author{
publiée à Lyon en 1582, \\ et premier ouvrage français entièrement consacré à l'odontologie
}

\begin{abstract}
on auteur, Urbain Hémard, un chirurgien de Rodez (vers 1548-1592), l'a écrit pour son $\checkmark$ protecteur, le cardinal Georges d'Armagnac (1501-1585), qui, souffrant de violentes rages de dents, lui demanda les causes \& raisons d'une si forte douleur.
\end{abstract}

Pour le lecteur non averti, la découverte de cet ouvrage rarissime est une surprise réjouissante. II découvrira les questions qui se posaient alors : si les dents sont des os plus durs que les autres, si elles ont sentiment, comment naissent et sortent les fendantes ou tranchantes, les canines ou dents de chien ou encore œillères et les machelieres ou marteaux. II appréciera les judicieux conseils pour assurer une bonne dentition aux enfants. Il frémira à la description des maladies et de la douleur omniprésente et doutera de l'arsenal thérapeutique fait d'astringents et de narcotiques remèdes ou estupefactifs pour hébéter le sentiment. Il apprendra aussi que faute d'arracheurs de dent, qui se trouvent exprès aux grandes villes et menteurs, parce qu'ils promettent indifféremment heureuse yssue de toutes choses, le chirurgien doit être exercé à cette œuvre. Il saura que les aymorragies post-extraction étaient souvent mortelles et conviendra volontiers que la guérison de la douleur des Dents par billets \& par charmes est affaire de suggestion. II mesurera la crudité des situations à laquelle tout homme pratiquant les arts de guérir était alors confronté. La Recherche est aussi un document socio-historique de cette deuxième moitié du $\mathrm{XvI}^{\mathrm{e}}$ siècle témoignant de la dureté et de la précarité de la vie sans cesse menacée.

Outre l'intégralité du traité original, le lecteur trouvera dans l'ouvrage plusieurs mises au point de spécialistes éclairant d'un jour nouveau la personnalité d'Urbain Hémard et sa place dans I'histoire médicale :

Pierre LANÇON - Le destin mouvementé d'un chirurgien ruthénois au XVIe siècle.

Micheline RUEL-KELLERMANN - La Recherche : un ouvrage marquant dans la littérature médicale du $\mathrm{xvle}$ siècle.

Jean-Pierre BÉNÉZET - Le médicament dans la Recherche d'Urbain Hémard.

Bernard CLUZEL - Instrumentation et art dentaire au XVIe siècle.

Nicole LEMAITRE - Le cardinal et les conseils avisés, ou du bon usage du français.

Ouvrage au format $16 \times 24 \mathrm{~cm}, 200$ pages, illustrations en noir et blanc couverture couleur gaufrée

( $25 €$ - port $4,50 €$ par exemplaire)

Société des lettres, sciences et arts de l'Aveyron - BP 125 - 12001 RODEZ Cedex

Tel./Fax 0565427593 